\title{
A case-control study on perinatal outcome in neonates with cord around the neck in a tertiary care hospital, Hubli, Karnataka
}

\author{
Donimath Kasturi $^{1}$, Smruthi Marathe $^{1}{ }^{*}$, Nekar Manjunath ${ }^{2}$
}

\author{
${ }^{1}$ Department of Obstetrics \& Gynecology, KIMS, Hubli, Karnataka, India \\ ${ }^{2}$ Department of Community Medicine, KIMS, Hubli, Karnataka, India
}

Received: 13 December 2013

Accepted: 6 January 2014

\section{*Correspondence:}

Dr. Smruthi Marathe,

E-mail: drsmruthi21@gmail.com

(O) 2014 Kasturi D et al. This is an open-access article distributed under the terms of the Creative Commons Attribution Non-Commercial License, which permits unrestricted non-commercial use, distribution, and reproduction in any medium, provided the original work is properly cited.

\begin{abstract}
Background: Intra-partum finding of umbilical cord around the baby's neck is a common finding, seen in about a third of normal deliveries. This nuchal cord has often been the scapegoat for complicated deliveries with adverse outcome like asphyxia or stillbirth. The aim of the study is to compare the perinatal outcome in full term vaginal deliveries in babies with cord around the neck and without cord intra-partum.

Methods: A total of 300 full term deliveries in labour were studied, with 150 cases with a single or multiple loops of cord and another 150 cases without cord around the neck. The cases were chosen randomly in a study period between August 2012 and May 2013. Perinatal outcome is analysed in the new-borns by means of APGAR score of the neonate at 1 and 5 minutes, NICU admission and complications till day 7 of life.

Results: $74 \%$ of the 150 deliveries with the cord around the neck were uneventful with clear liquor status and normal FHR variability. $22 \%$ of the cases had thin meconium stained liquor and $4 \%$ were associated with thick meconium stained liquor. The difference in the Apgar score at 1 and 5 minutes between the study and the control group was statistically not significant. Also there was no statistically significant difference in NICU admissions and neonatal morbidity.

Conclusions: The study has concluded that the finding of cord round the neck in fetuses does not cause any specific adverse outcome in properly monitored labor. The study has not shown any stillbirth that could be attributed to the cord. Hence, mention of a nuchal cord in the ultrasound reports does not warrant panic and a safe vaginal delivery can be contemplated successfully.
\end{abstract}

Keywords: Nuchal cord, APGAR, Asphyxia, FHR

\section{INTRODUCTION}

Intra-partum finding of umbilical cord around the baby's neck is a common finding, seen in about a third of normal deliveries. ${ }^{1}$ This nuchal cord has often been the scapegoat for complicated deliveries with adverse outcome like asphyxia or stillbirth. With the increasing use of color Doppler in Ultrasound, nuchal cord has become a part of the report. The finding of either a single or multiple loops of cord around the neck increases with increasing gestational age and with long cords. It occurs in 2 types $^{2}$ Type A meaning that the loop/s of cord is/are loose around the baby's neck and Type B meaning that the loop/s is/are tight. Nuchal cord usually causes mild to moderate variable decelerations during the second stage of labour due to cord compression. There is an acute fall in FHR with a rapid downslope with a variable recovery phase. The decelerations resemble the letters ' $U$ ', ' $V$ ' or ' $\mathrm{W}$ ' and do not bear a constant relationship with uterine contractions. ${ }^{3}$ Pressure on the cord initially occludes the umbilical vein which results in acceleration and indicates a healthy response. This is followed by occlusion of the umbilical artery which causes a sharp downslope. Finally, the recovery phase is due to the relief of the compression and the sharp turn to baseline, which may be followed by another healthy brief acceleration. 
Variable decelerations are classified according to their depth and duration as,

Mild - Depth above 80bpm lasting less than 60 seconds

Moderate - Depth 70-80bpm lasting 30-60 seconds

Severe - Depth less than 70bpm lasting more than 60 seconds

Variable decelerations are generally associated with a favourable outcome. However, persistent severe decelerations with loss of beat-to-beat variability are nonreassuring and indicate fetal hypoxia and acidosis. Variable decelerations on CTG:

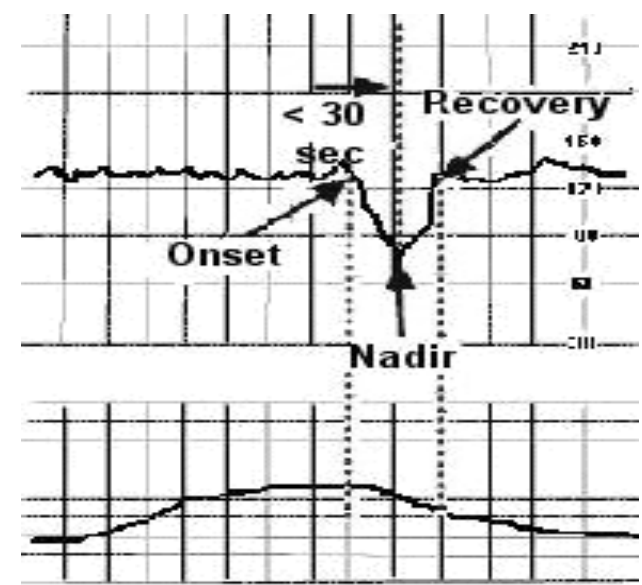

Figure 1: Variable decelerations on CTG. ${ }^{4}$

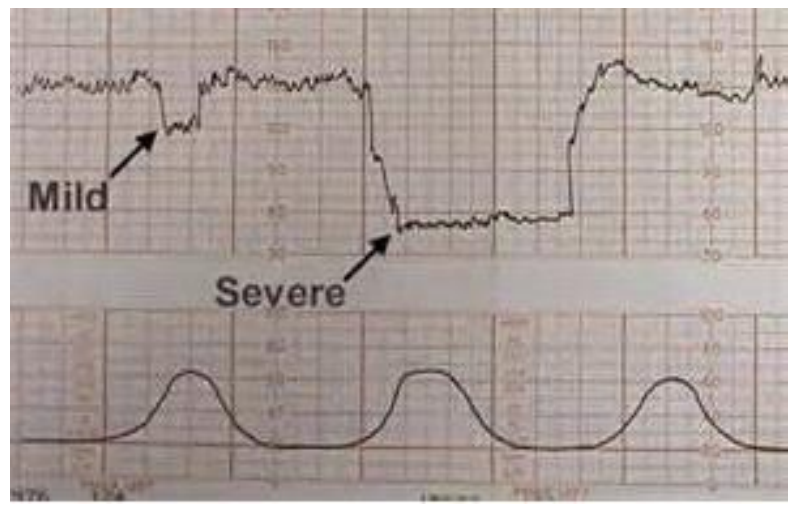

Figure 2: Mild and severe variable decelerations. ${ }^{5}$

An APGAR score of 3 or less at 5 minutes of birth is considered an essential requirement for the diagnosis of perinatal asphyxia. However, a persistently low score alone is not a specific indicator of intra-partum insult. The AAP and ACOG define perinatal asphyxia when all the following criteria are met. ${ }^{6}$

- $\quad$ Profound metabolic or mixed acidemia

- APGAR Score 0-3 at 5 minutes of birth

- Neonatal Encephalopathy
- Multiple Organ Involvement (kidney, lung, liver, heart, intestines)

\section{Aims and objectives}

The comparative study is conducted at Karnataka Institute of Medical Sciences, Hubli in randomly selected cases and controls matched for gravidity with the following objectives:

- To study the occurrence of stillbirths in the cases

- To assess intra-partum asphyxia which increases perinatal morbidity and mortality

\section{METHODS}

The study is conducted from September 2012 to May 2013 at KIMS, Hubli.

\section{Inclusion criteria}

Singleton pregnancies of $37-42$ weeks gestation with cephalic presentation are selected randomly.

\section{Exclusion criteria}

Patients with any risk factors like Anemia, PIH, Congenital anomaly, Preterm delivery, Chorioamnionitis, Multi-fetal gestation etc. which can independently cause adverse perinatal and neonatal outcome and can act as confounding factors are excluded from the study.

150 cases with loop/s of cord around the neck were retrospectively followed to note the gravidity, type of FHR variability, liquor status, number of tight/loose loops of cord around the neck and followed-up for the APGAR at 1 and 5 minutes, NICU admission and the health of the baby for 7 days of life. Babies with a weak cry at birth or with meconium-stained amniotic fluid were immediately handed over to the pediatrician for resuscitation. The babies discharged prior to 7 days postdelivery were followed-up on telephone with the mothers and reviewed on $7^{\text {th }}$ day to look for the health status of the baby.

150 randomly chosen full term singleton vaginal deliveries without any risk factors served as controls for a comparison in the outcomes of delivery. Primary outcomes involved stillbirths and asphyxia leading to NICU admission. Secondary outcomes involved NICU admission due to other causes like meconium aspiration, hyperbilirubinemia, transient tachypnea, sepsis, respiratory distress etc. in both cases and controls.

\section{Statistical analysis}

Ratio, proportion are used to show the variability of data and Categorical variables were compared by Fischer's exact test. 


\section{RESULTS}

A total of 300 deliveries were studied, with 150 being the cases.

In the study group, $74 \%$ out of 150 cases had clear liquor, $22 \%$ had thin meconium stained liquor and $4 \%$ had a thick meconium stained liquor (Table 1). The meconium in these cases was detected in the $2^{\text {nd }}$ stage of labor in primigravidae when assisted vaginal deliveries were contemplated, and in the late active phase of labor in multigravidae when labor was accelerated to hasten delivery. All the cases with meconium were associated with normal FHR variability on CTG and occasional reversible variable decelerations in the $2^{\text {nd }}$ stage of labor due to which vaginal delivery was allowed. All babies with meconium staining were attended by the paediatrician at birth and only 3 of the 39 neonates required intervention at 1 minute.

Table 1: Liquor status.

\begin{tabular}{|lll|}
\hline & Frequency & Percent \\
\hline Number & 150 & 100 \\
\hline Clear & 111 & 74 \\
\hline Thick meconium & 6 & 4 \\
\hline Thin meconium & 33 & 22 \\
\hline Total & 300 & 100.0 \\
\hline
\end{tabular}

There were no cases of acute fetal distress. APGAR $<7$ at 1 minute was seen in $2 \%$ of cases in the study group and $3.3 \%$ cases in the control group. But this difference is statistically not significant.

Table 2: APGAR score of neonates at 1 min.

\begin{tabular}{|lllll|}
\hline APGAR score at & \multicolumn{2}{c|}{ Study group } & \multicolumn{2}{c|}{ Control group } \\
$\mathbf{m i n}$ & No. & $\mathbf{\%}$ & No. & $\mathbf{\%}$ \\
\hline APGAR score $<6$ & 0 & 0 & 2 & 1.4 \\
\hline APGAR score $>7$ & 150 & 100 & 148 & 98.6 \\
\hline
\end{tabular}

Chi Square value: 2.013

Fisher's exact test $\mathrm{P}$ value: 0.498 (not significant)

APGAR $<7$ at 5 minutes was seen in $1.3 \%$ cases in the study group and $0 \%$ cases in the control group. This difference is also not statistically significant.

Table 3: APGAR score of neonates at $5 \mathrm{~min}$.

\begin{tabular}{|lllll|}
\hline APGAR score at & \multicolumn{2}{c|}{ Study group } & \multicolumn{2}{c|}{ Control group } \\
$\mathbf{m i n}$ & No. & $\mathbf{\%}$ & No. & \% \\
\hline APGAR score $<6$ & 0 & 0 & 1 & 0.7 \\
\hline APGAR score $>7$ & 150 & 100 & 149 & 99.3 \\
\hline
\end{tabular}

Chi square value: 1.003

(Fisher's exact test) P Value: 1.000 (Not Significant).
Among the NICU admissions, $1.2 \%$ neonates had birth asphyxia in the study group as compared to $0.6 \%$ in controls, $\mathrm{P}$ value being 1 . One new-born each in the study and control group was admitted for LBW and SGA with LBW respectively. Equal number of new-borns in study and control group i.e., $1.3 \%$ had TTN. MAS was not seen in any of the new-borns of either group. No mortality was seen in the study group, presumed to be complicated cases with loops of cord around the neck.

Table 4: Neonatal morbidity.

\begin{tabular}{|l|llll|}
\hline \multirow{2}{*}{$\begin{array}{l}\text { Perinatal morbidity } \\
\text { observed }\end{array}$} & Cases & \multicolumn{3}{c|}{ Controls } \\
\hline Birth asphyxia- mild & 1 & 0.6 & 0 & 0.6 \\
\hline Low birth weight & 1 & 0 & 0 & 0.6 \\
\hline SGA with LBW & 0 & 0 & 1 & 0.6 \\
\hline TTN & 2 & 1.3 & 2 & 1.3 \\
\hline
\end{tabular}

Cross tabulation was done between the incidence of complications in the study group and the control group. Chi Square value: 0.416

Fisher's exact test 'P' value: 1.00 (Statistically insignificant)

\section{DISCUSSION}

With increasing use of Color Doppler in USG and increasing reporting of a nuchal cord, increased concern remains in obstetricians about the possibility of birth asphyxia and neonatal mortality, nevertheless fear of litigations. It in indeed frightening for the pregnant woman and her family to know of a loop of cord round the baby's neck which gives them a mental image of strangulation!!! All this leads to a tendency to opt for an Elective LSCS by both obstetricians and the patient and her family.

James et al. ${ }^{7}$ at Cleveland, Ohio conducted a prospective subject, examiner and physician blinded study. They examined fetuses serially at 24-26, 30-32 and 36-38 weeks gestation and during labour and delivery and concluded that nuchal cord is not associated with clinical evidence of fetal compromise before labour.

Sheiner et al. ${ }^{8}$ conducted a retrospective population based study of deliveries from 1988-2003 at Soroko University Medical Center in Israel and concluded that nuchal cord has no adverse perinatal outcome.

Shrestha NS et al. ${ }^{9}$ at Kathmandu Medical College studies the incidence, intra-partum complications and perinatal outcomes in cases with nuchal cord by a prospective, cross-sectional, comparative study from March to September 2006 of 512 deliveries and found that FHR irregularities and meconium staining were increased in the nuchal cord group but were statistically not significant.

Assimakopoulus E et al. ${ }^{10}$ at Papageorgiou General Hospital in Greece evaluated the clinical relevance of 
nuchal cord in 352 normal singleton pregnancies with vertex presentation prospectively with real time ultrasound at 37-39 weeks. Health care workers were blinded about the nuchal cord. There were increased rates of operative vaginal deliveries and caesarean sections in nulliparae.

Jauniaux E et al. $^{11}$ at Free University at Brussels, Belgium compared perinatal data of 550 fetuses with a nuchal cord at delivery to a control group matched for gestational age, maternal age and parity. There was a higher incidence of APGAR score less than 7 at 1 minute, meconium stained amniotic fluid, emergency caesarean section, need for neonatal resuscitation and of admission to the NICU in the nuchal cord group compared with the controls.

Vaginal delivery is safe in cases with cord around the neck as in the cases without cord. Regular monitoring of the FHR by intermittent auscultation initially, early ARM, continuous FHR monitoring by CTG from the late active phase onwards prove to be of immense help to contemplate a safe vaginal delivery. Non-persistent variable decelerations do not imply fetal jeopardy and can be managed expectantly by rest in left lateral position, Oxygen and by accelerating labor. Assisted vaginal deliveries in the $2^{\text {nd }}$ stage of labor are valuable.

\section{CONCLUSIONS}

Finding a single or multiple loops of cord around the neck of the baby is physiological and is seen in $25 \%$ of deliveries. ${ }^{12}$ It is more associated with a long cord. There is no comparable difference in the outcomes in cases with cord and without cord around the neck and hence, vaginal delivery can be safely contemplated in well monitored labours.

\section{Funding: No funding sources}

Conflict of interest: None declared

Ethical approval: The study was approved by the institutional ethics committee

\section{REFERENCES}

1. James F. Clapp III, William Stephenchak, Kazumasa Hashimoto, Hugh Ehrenberg, Beth Lopez. The natural history of antenatal nuchal cords. Am J Obstet Gynecol. 2003;189:488-93.

2. Jason H. Collins. Nuchal cord type A and type B. Am J Ag. 1997;177:94.
3. Amir Sweha, Trevor W. Hacker, Jim Nuovo. Interpretation of the electronic fetal heart rate during labour. Am Fam Physician. 1999 May;59(9):24872500.

4. American College of Obstetrics and Gynecology. Task Force on Neonatal Encephalopathy and Cerebral Palsy. American Academy of Pediatrics. Neonatal Encephalopathy and Cerebral Palsy: Defining the Pathogenesis and Pathophysiology. Edited by Washington, DC, American College of Obstetricians and Gynecologists, 2003. Available at: http://www.acog.org/.

5. Perinatology. Intrapartum fetal heart rate monitoring and variable decelerations, 2013. Available at: www.perinatology.com. Accessed 26 November 2013.

6. Ttuhsc. Intrapartum fetal heart rate monitoring and variable decelerations, 2013. Available at: www.ttuhsc.com. Accessed 26 November 2013.

7. James F. Clapp III, William Stephenchak, Kazumasa Hashimoto, Hugh Ehrenberg, Beth Lopez. The natural history of antenatal nuchal cords. Am J Obstet Gynecol. 2003;189:488-93.

8. Shiener E, Abramowicz JS, Levy A, Silberstein T, Mazor M, Hershkovitz R. Nuchal cord is not associated with adverse perinatal outcome. Arch Gynecol Obstet. 2006 May;274(2):81-3.

9. Shrestha NS, Singh N. Nuchal cord and perinatal outcome. Kathmandu Univ Med J. 2007 JulSep;5(32):360-3.

10. Assimakopoulos E, Zafrakas M, Garmiris P, Goulis DG, Athanasiadis AP, Dragoumis K, Bontis J. Nuchal cord detected at ultrasound at term is associated with mode of delivery and perinatal outcome. Eur J Obstet Gynecol Reprod Biol. 2005 Dec;123(2):188-92.

11. Jauniaux E, Ramsay B, Peellaerts C, Scholler Y. Perinatal features of pregnancies complicated by nuchal cord. Am J Perinatol. 1995 Jul;12(\$):255-8.

12. William's. Normal labor and delivery. In: F. Gary Cunningham, Kenneth J. Leveno, Steven L. Bloom, John C. Hauth, Dwight J. Rouse, Catherine Y. Spong, eds. William's Obstetrics. 23rd ed. New York, NY: McGraw-Hill; 2010: 397.

DOI: $10.5455 / 2320-1770 . i j r \operatorname{cog} 20140321$

Cite this article as: Kasturi $D$, Marathe $S$, Manjunath

N. A case-control study on perinatal outcome in neonates with cord around the neck in a tertiary care hospital, Hubli, Karnataka. Int J Reprod Contracept Obstet Gynecol 2014;3:105-8. 